\title{
Cyclic Čech-Hochschild bicomplex
}

\author{
Jan Kubarski
}




\title{
CYCLIC ČECH-HOCHSCHILD BICOMPLEX
}

\author{
JAN KUBARSKI
}

\begin{abstract}
In classical differential topology and geometry, the double Čech-de Rham complex for a good covering $\mathfrak{U}$ of $M$ corresponds to the single de Rham complex. When passing to noncommutative geometry on smooth manifolds (i. e. to noncommutative geometry of the algebra $C^{\infty}(M)$ of smooth functions on $\left.M\right)$, the single Connes complex is the counterpart of the single de Rham complex. We can ask: which bicomplex corresponds to the single Connes complex in noncommutative geometry of smooth manifolds? We give an answer to this question defining the Cyclic Čech-Hochschild bicomplex for a good covering of a smooth manifold. We pose fundamental problems on the total homology of this bicomplex.
\end{abstract}

2010 Mathematics Subject Classification: 58B34, 16E40, 55U15, 57R20

Keywords: Double Čech-de Rham complex, good covering, noncommutative geometry on smooth manifolds, single Connes complex, cyclic complex, single de Rham complex, cyclic ČechHochschild bicomplex, Čech cohomology, cocycle, local formulas for characteristic classes, Hochschild homology, Cyclic homology, Chern character

\section{1. ČECH - DE RHAM BICOMPLEX FOR A GOOD COVERING}

In differential geometry and topology of smooth manifolds, a fundamental role is played by the de Rham complex $\left(\Omega^{*}(M), d\right), \operatorname{dim} M=n$,

$$
\Omega^{0}(M) \stackrel{d}{\longrightarrow} \ldots \stackrel{d^{q-1}}{\longrightarrow} \Omega^{q}(M) \stackrel{d^{q}}{\longrightarrow} \Omega^{q+1}(M) \stackrel{d^{q+1}}{\longrightarrow} \ldots \stackrel{d}{\longrightarrow} \Omega^{n}(M) \rightarrow 0
$$

of differential forms and the de Rham differential operator $d=d_{d R}$. The cohomology of this complex is the de Rham cohomology of the manifold $M$,

$$
\mathbf{H}_{D R}^{q}(M)=\operatorname{ker} d^{q} / \operatorname{Im} d^{q-1} .
$$

The Čech - de Rham bicomplex for a good covering $\mathfrak{U}$ of $M$ corresponds to the single de Rham complex,

$$
\left(C^{*}\left(\mathfrak{U}, \Omega^{*}\right), \delta, \tilde{d}\right)
$$


(over the first quadrant) where $C(\mathfrak{U}, \Omega)=\left\{C^{p}\left(\mathfrak{U}, \Omega^{q}\right)\right\}_{p \geq 0, q \geq 0}, \delta=\delta_{\check{C}}$ is the Čech differential and $\tilde{d}=(-1)^{p} d$ on $C^{p}\left(\mathfrak{U}, \Omega^{*}\right), \delta \tilde{d}+\tilde{d} \delta=0$,

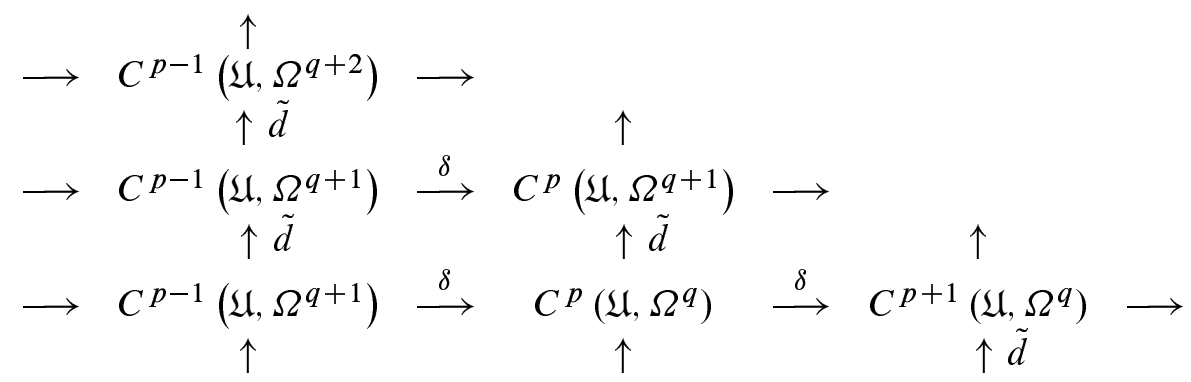

The total differential $D=\delta+\tilde{d}: C^{(r)} \rightarrow C^{(r+1)}$ is homogeneous of degree +1 with respect to the total gradation

$$
C^{(r)}=\bigoplus_{p+q=r} C^{p}\left(\mathfrak{U}, \Omega^{q}\right)
$$

along the lines $p+q=$ const.

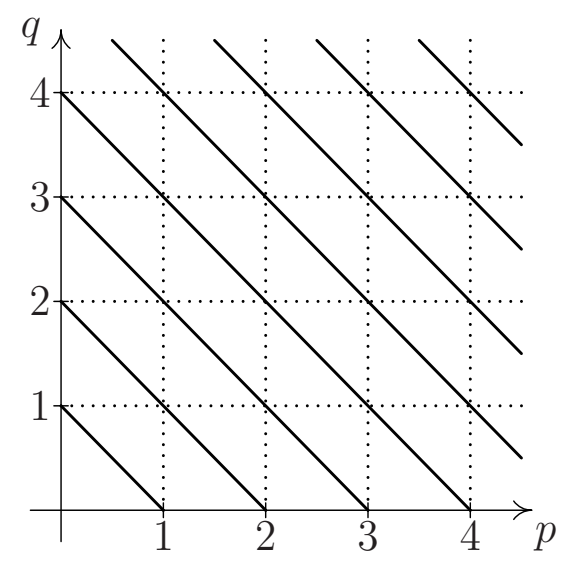

FIGURE 1. Total gradation along $p+q=$ const.

In spite of the fact that rows and columns of the double Čech - de Rham bicomplex are exact, the total cohomology of this bicomplex is, on the one hand, isomorphic to the de Rham cohomology and on the other hand to the Čech cohomology:

$$
\mathbf{H}_{D}^{(r)}=\mathbf{H}_{D R}^{r}(M)=\check{\mathbf{H}}^{r}(M) .
$$


Therefore, the characteristic classes of $M$ (and more generally of any vector bundle or any $G$-principal fibre bundle) could be determined by cochain forms defined locally from the double Čech - de Rham bicomplex. See the results by Bott [1], Bott and Tu [2, pp. 304-305], and Sharygin [11].

The Bott, Bott and Tu, and Sharygin approach consists in producing local formulas in the Čech-de Rham bicomplex by using transition functions $g_{i j}: U_{i} \cap U_{j} \rightarrow G$ (forming a cocycle, i. e., $g_{i j} g_{j k}=g_{i k}$ ) with values in a suitable Lie group $G$. The transition functions $g_{i j}$ are obtained from a system of local trivializations of a suitable principal fibre bundle.

All such formulas for characteristic classes obtained for chains defined for a good covering are called local formulas for characteristic classes.

And what is the corresponding object in noncommutative geometry? The counterpart of the de Rham complex is the Connes complex for an associative algebra $A$ with a unit over the field $K=\mathbb{R}$ or $\mathbb{C}$, which lies at the foundations of noncommutative geometry [3-5].

\section{Single CONNES COMPLEX}

Put crudely, the (abstract) Connes complex consists of cyclic Hochschild chains and bar differentials

$$
\left(C_{*}(A) / \operatorname{Im}\left(1-T_{*}\right),\left[b_{*}\right]\right) \cong\left(C_{*}^{\lambda}(A)=\operatorname{ker}\left(1-T_{*}\right), b_{*}^{\prime}\right)
$$

where $C_{q}(A)=\bigotimes^{q+1}(A)$, which determines the cyclic homology $\mathbf{H}_{*}^{\lambda}(A)$ [10]. For a review of abstract Hochschild and Cyclic Homology, see [8, 12].

Now, we turn to noncommutative geometry on smooth manifolds, i. e. to the algebra $A=C^{\infty}(M)$ of smooth functions on $M$.

The relation between the above-stated abstract Hochschild theory of an arbitrary algebra $A$ and the "smooth theory" for manifolds uses the tensor power $\widehat{\otimes}{ }^{q+1} C^{\infty}(M)$ in the category of topological linear spaces, following the introduction of the Fréchet topology in the algebra of smooth functions $C^{\infty}(M)$ (see, for example, [6]). We have the following identification of $\widehat{\otimes}^{q+1} C^{\infty}(M)$ with the space of smooth functions on the cartesian product $M^{q+1}$ :

$$
\begin{gathered}
\widehat{\bigotimes}^{q+1} C^{\infty}(M)=C_{q}\left(C^{\infty}(M)\right):=\left\{\phi^{q}: M^{q+1} \rightarrow \mathbb{R}\right\}, \\
f_{0} \otimes \ldots \otimes f_{q}\left(x_{0}, \ldots, x_{q}\right)=f_{0}\left(x_{0}\right) \cdot \ldots \cdot f_{q}\left(x_{q}\right) .
\end{gathered}
$$

The above-said allows us to apply all the cyclic homology differentials like $b, b^{\prime}$, $B$ etc. to Hochschild $q$-chains $C_{q}\left(C^{\infty}(M)\right)$. The bar differential

$$
b_{q}^{\prime}: C_{q}\left(C^{\infty}(M)\right) \rightarrow C_{q-1}\left(C^{\infty}(M)\right)
$$


is defined by the formula

$$
\left(b_{q}^{\prime}\left(\phi^{q}\right)\right)\left(x_{0}, \ldots, x_{q-1}\right)=\sum_{i=0}^{q-1}(-1)^{i} \phi^{q}\left(x_{0}, \ldots, x_{i}, x_{i}, \ldots, x_{q-1}\right) .
$$

We introduce the graded cyclic permutation

$$
\begin{gathered}
T_{q}: C_{q}\left(C^{\infty}(M)\right) \rightarrow C_{q}\left(C^{\infty}(M)\right), \\
T_{q}\left(\phi^{q}\right)\left(x_{0}, \ldots, x_{q}\right)=(-1)^{q} \phi^{q}\left(x_{1}, \ldots, x_{q}, x_{0}\right) .
\end{gathered}
$$

Definition 2.1. A $q$-chain $\phi^{q}$ is called cyclic if $T_{q}\left(\phi^{q}\right)=\phi^{q}$, i.e.

$$
\phi^{q} \text { is cyclic } \equiv \phi^{q} \in \operatorname{ker}\left(1-T_{q}\right) .
$$

The space of cyclic $q$-chains is denoted by $C_{q}^{\lambda}\left(C^{\infty}(M)\right)$.

The space of cyclic chains is stable under the bar differential:

$$
b^{\prime}\left(C_{q}^{\lambda}\left(C^{\infty}(M)\right)\right) \subset C_{q-1}^{\lambda}\left(C^{\infty}(M)\right) .
$$

Definition 2.2. The complex $\left(C_{*}^{\lambda}\left(C^{\infty}(M)\right), b^{\prime}\right)$ is called the cyclic complex or Connes complex of the manifold $M$. Its $k$ th homology group is denoted by $\mathbf{H}_{k}^{\lambda}\left(C^{\infty}(M)\right)$ :

$$
\mathbf{H}_{k}^{\lambda}\left(C^{\infty}(M)\right):=\mathbf{H}_{k}\left(C_{*}^{\lambda}\left(C^{\infty}(M)\right), b^{\prime}\right) .
$$

The homology of this complex was given by Connes (for compact manifolds) and by Teleman (for paracompact manifolds). See also [9].

Theorem 2.3 (Connes-Teleman theorem). For a paracompact manifold $M$, we have

$$
\mathbf{H}_{0}^{\lambda}\left(C^{\infty}(M)\right)=\Omega^{0}(M)=C^{\infty}(M),
$$

and for $i \geq 1$,

$\mathbf{H}_{2 i}^{\lambda}\left(C^{\infty}(M)\right)$

$=\mathbf{H}^{0}(M) \oplus \mathbf{H}^{2}(M) \oplus \mathbf{H}^{4}(M) \oplus \cdots \oplus \mathbf{H}^{2 i-2}(M) \oplus \Omega^{2 i}(M) / d\left[\Omega^{2 i-1}(M)\right]$,

$\mathbf{H}_{2 i+1}^{\lambda}\left(C^{\infty}(M)\right)$

$=\mathbf{H}^{1}(M) \oplus \mathbf{H}^{3}(M) \oplus \mathbf{H}^{5}(M) \oplus \cdots \oplus \mathbf{H}^{2 i-1}(M) \oplus \Omega^{2 i+1}(M) / d\left[\Omega^{2 i}(M)\right]$.

In particular, if $\operatorname{dim} M$ is even, $\operatorname{dim} M=2 m$, then

$$
\begin{aligned}
\mathbf{H}_{2 m+2}^{\lambda}\left(C^{\infty}(M)\right) & =\mathbf{H}_{2 m+4}^{\lambda}\left(C^{\infty}(M)\right)=\ldots \\
& =\mathbf{H}^{0}(M) \oplus \mathbf{H}^{2}(M) \oplus \mathbf{H}^{4}(M) \oplus \cdots \oplus \mathbf{H}^{2 m}(M),
\end{aligned}
$$

while if $\operatorname{dim} M$ is odd, $\operatorname{dim} M=2 m+1$, then

$$
\begin{aligned}
\mathbf{H}_{2 m+2}^{\lambda}\left(C^{\infty}(M)\right) & =\mathbf{H}_{2 m+4}^{\lambda}\left(C^{\infty}(M)\right)=\ldots \\
& =\mathbf{H}^{0}(M) \oplus \mathbf{H}^{2}(M) \oplus \mathbf{H}^{4}(M) \oplus \cdots \oplus \mathbf{H}^{2 m}(M) .
\end{aligned}
$$


Therefore, the whole algebra of characteristic classes (Chern character) is sitting in one homology space, $\mathbf{H}_{2 m+2}^{\lambda}\left(C^{\infty}(M)\right)$.

Conclusion 2.4. If $U \subset M$ is a contractible open subset $\left(U \cong \mathbb{R}^{m}\right)$, then $\mathbf{H}^{k}(U)=$ 0 for $k>0, \mathbf{H}^{0}(U)=\mathbb{R}$, therefore

$$
\begin{aligned}
\mathbf{H}_{2 i}^{\lambda}\left(C^{\infty}(U)\right) & =\mathbb{R} \oplus\left(\Omega^{2 i}(U) / d\left[\Omega^{2 i-1}(U)\right]\right) \\
& =\mathbb{R} \oplus\left(\Omega^{2 i}(U) / \operatorname{ker} d^{2 i}\right)=\mathbb{R} \oplus \operatorname{Im} d^{2 i}, i \geq 1, \\
\mathbf{H}_{0}^{\lambda}\left(C^{\infty}(U)\right) & =C^{\infty}(U), \\
\mathbf{H}_{2 i-1}^{\lambda}\left(C^{\infty}(U)\right) & =\left(\Omega^{2 i-1}(U) / \operatorname{ker} d_{2 i-1}\right)=\operatorname{Im} d_{2 i-1} .
\end{aligned}
$$

\section{3. ČECH-HOCHSCHILD BICOMPLEX FOR A GOOD COVERING}

In classical differential topology and geometry, the single de Rham complex corresponds to the double Čech - de Rham complex for a good covering $\mathfrak{U}$ of $M$. And what is the case in noncommutative geometry of smooth manifolds?

We give an answer to this question. A double complex counterpart in noncommutative geometry of smooth manifolds has been defined by N. Teleman.

During his visit to the Institute of Mathematics of the Lodz University of Technology in December 2009 at the invitation of the author, N. Teleman suggested the definition of the Cyclic Čech-Hochschild bicomplex for a good covering of a manifold, which corresponds to the single Connes complex, and posed the problem of computing its total homology in connection with the problem of computing characteristic classes.

Definition 3.1 (Čech-Hochschild bicomplex). By the Čech-Hochschild bicomplex for a good covering $\mathfrak{U}$ of $M$, we mean a bicomplex (over the first quadrant) of vector spaces

$$
\left(\left\{\check{C} H_{p, q}^{\lambda}(\mathfrak{U}, \mathbb{R})\right\}_{p \geq 0, q \geq 0}, \tilde{\delta}, b^{\prime}\right)
$$

where

(a)

$$
\begin{aligned}
\check{C} H_{p, q}(\mathfrak{U}, \mathbb{R}) & =\bigoplus_{\left(i_{0}, \ldots, i_{p}\right) \in I^{p+1}} C_{\left[i_{0}, \ldots, i_{p}\right], q}(\mathfrak{U}, \mathbb{R}), \\
C_{\left[i_{0}, \ldots, i_{p}\right], q}(\mathfrak{U}, \mathbb{R}) & =\left\{\phi_{i_{0} \ldots i_{p}}^{q}:\left(U_{i_{0} \ldots i_{p}}\right)^{q+1} \rightarrow \mathbb{R}\right\},
\end{aligned}
$$

(b) $b^{\prime}$ is the bar differential for $p \geq 0, q \geq 1$,

$$
\begin{gathered}
b_{p, q}^{\prime}: \check{C} H_{p, q}(\mathfrak{U}, V) \rightarrow \check{C} H_{p, q-1}(\mathfrak{U}, V), \\
\left(b_{p, q}^{\prime}\left(\phi_{p}^{q}\right)\right)_{i_{0}, \ldots, i_{p}}\left(x_{0}, \ldots, x_{q-1}\right)=\sum_{i=0}^{q-1}(-1)^{i} \phi_{i_{0} \ldots i_{p}}^{q}\left(x_{0}, \ldots, x_{i}, x_{i}, \ldots, x_{q-1}\right) .
\end{gathered}
$$


(c) $\tilde{\delta}$ is a sign modification of the Čech differential for $p, q \geq 0$,

$$
\begin{gathered}
\delta^{p, q}: \check{C} H_{p, q}(\mathfrak{U}, V) \rightarrow \check{C} H_{p+1, q}(\mathfrak{U}, V), \\
\delta^{p, q}\left(\phi^{q}\right)_{i_{0}, \ldots, i_{p+1}}=\sum_{r=0}^{p+1}(-1)^{r} \phi_{i_{0}, \ldots, \hat{i_{r}}, \ldots, i_{p+1}}^{q}, \\
\tilde{\delta}^{p, q}=(-1)^{q} \delta^{p, q} .
\end{gathered}
$$

It is a standard calculation that $\tilde{\delta} b^{\prime}+b^{\prime} \tilde{\delta}=0$. We modify the sign of $\delta$, not of $b^{\prime}$, because in the standard $C C$-bicomplex in cyclic homology, we have " $-b^{\prime \prime}$ " everywhere and, technically, the $C C$-bicomplex is used in this theory.

We take the graded cyclic permutation

$$
\begin{gathered}
T_{p, q}: \check{C} H_{p, q}(\mathfrak{U}, \mathbb{R}) \rightarrow \check{C} H_{p, q}(\mathfrak{U}, \mathbb{R}), \\
T_{p, q}\left(\phi^{q}\right)_{i_{0}, \ldots, i_{p}}\left(x_{0}, \ldots, x_{q}\right)=(-1)^{q} \phi_{i_{0} \ldots i_{p}}^{q}\left(x_{1}, \ldots, x_{q}, x_{0}\right),
\end{gathered}
$$

and the cyclic functions

$$
\check{C} H_{p, q}^{\lambda}(\mathfrak{U}, \mathbb{R})=\left\{\phi \in \check{C} H_{p, q}(\mathfrak{U}, \mathbb{R}) ; T_{p, q}(\phi)=\phi\right\}=\operatorname{ker}\left(1-T_{q}\right) .
$$

The spaces of cyclic chains are stable under the bar and Čech differentials,

$$
b^{\prime}\left[\check{C} H_{p, q}^{\lambda}(\mathfrak{U}, \mathbb{R})\right] \subset \check{C} H_{p, q-1}^{\lambda}(\mathfrak{U}, \mathbb{R}), \quad \delta^{p, q}\left[\check{C} H_{p, q}^{\lambda}(\mathfrak{U}, \mathbb{R})\right] \subset \check{C} H_{p+1, q}^{\lambda}(\mathfrak{U}, \mathbb{R}) .
$$

Thus, we introduce a subcomplex $\left(\check{C} H^{\lambda}(\mathfrak{U}, \mathbb{R}), \tilde{\delta}, b^{\prime}\right), \check{C} H^{\lambda}=\operatorname{ker}(1-T)$, of the Čech-Hochschild bicomplex.

Definition 3.2 (Cyclic Čech-Hochschild bicomplex). By the name Cyclic ČechHochschild bicomplex, we mean the bicomplex $\left(\check{C} H^{\lambda}(\mathfrak{U}, \mathbb{R}), \tilde{\delta}, b^{\prime}\right)$,

$$
\begin{aligned}
& \stackrel{\tilde{\delta}}{\longrightarrow} \check{C} H_{p+2, q+1}^{\lambda}(\mathfrak{U}, \mathbb{R}) \longrightarrow \\
& \downarrow b^{\prime} \quad \downarrow b^{\prime}
\end{aligned}
$$

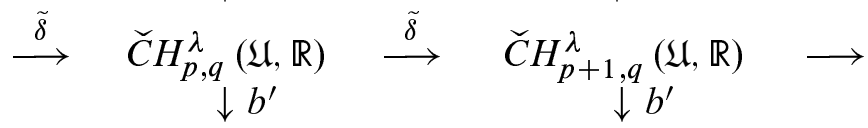

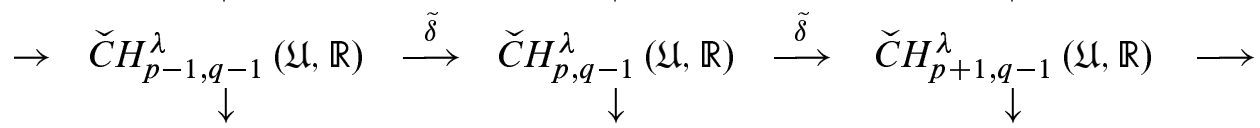

The total differential

$$
D=\tilde{\delta}+b^{\prime}: C^{[r]} \rightarrow C^{[r+1]}
$$


is homogeneous of degree +1 with respect to the total gradation

$$
C^{[r]}=\prod_{p-q=r} \check{C} H_{p, q}^{\lambda}(\mathfrak{U}, \mathbb{R})
$$

along the lines $p-q=$ const

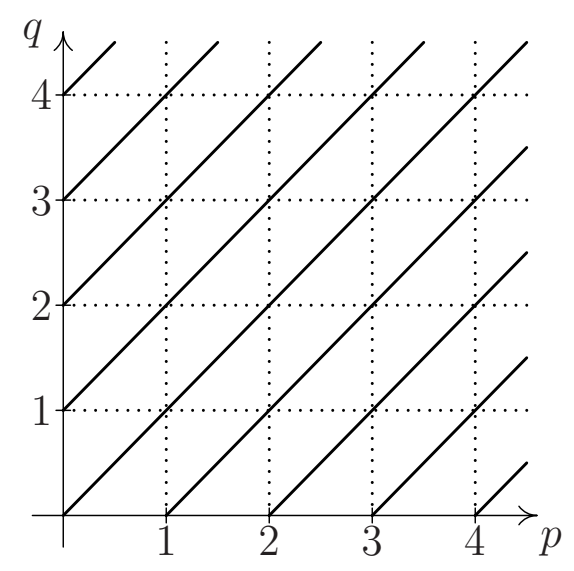

FIGURE 2. Total gradation along the lines $p-q=$ const

(we observe that on each line $p-q=$ const, we have infinitely many vector spaces and we must use the cartesian product of vector spaces, and not the direct sum; see [7, Th. 3.2]). Since $D^{2}=0$, we can define the total homology

$$
\mathbf{H}^{[r]}\left(\breve{C} H^{\lambda}(\mathfrak{U}, \mathbb{R})\right) \text {. }
$$

First main problem. Calculate the total homology of the Cyclic $\check{C} e c h-H o c h s c h i l d$ bicomplex.

We observe that the homology of the columns of the Cyclic Čech-Hochschild bicomplex is the homology of the suitable Connes complex for contractible sets $U_{i_{0} \ldots i_{p}}=U_{i_{0}} \cap \ldots \cap U_{i_{p}}$, therefore, the above mentioned Connes-Teleman theorem on homology of the complex $C_{*}^{\lambda}\left(C^{\infty}\left(U_{i_{0} \ldots i_{p}}\right)\right)$ is very useful.

It turns out that the total homology of the cyclic Cech-Hochschild bicomplex $\left(\breve{C} H_{p, q}^{\lambda}, \tilde{\delta}, b^{\prime}\right)$ is very rich [7], so it is reasonable to look for representations of characteristic classes in the homology of this bicomplex.

Second main problem. Find a Cyclic Čech-Hochschild chain which represents characteristic classes.

This problem will be discussed elsewhere. 


\section{REFERENCES}

[1] R. Bott, "On the Chern-Weil homomorphism and the continuous cohomology of Lie groups," Advances in Math., vol. 11, pp. 289-303, 1973.

[2] R. Bott and L. Tu, Differential Forms in Algebraic Topology, ser. GTM. Springer, 1982.

[3] A. Connes, "Noncommutative differential geometry," Publ. Math. IHES, vol. 62, pp. 257-360, 1985.

[4] A. Connes, Noncommutative Geometry. Academic Press, 1994.

[5] A. Connes and H. Moscovici, "Cyclic cohomology, the novikov conjecture and hyperbolic groups," Topology, vol. 29, pp. 345-388, 1990.

[6] C.-O. Ewald, Hochschild Homology and de Rham Cohomology of Stratifolds. Heidelberg, 2002.

[7] J. Kubarski, "Topology of the Cyclic Čech-Hochschild bicomplex," in preparation.

[8] J. Kubarski and N. Teleman, Geometry and Topology of Manifolds: The Mathematical Legacy of Charles Ehresmann. Banach Center Publ., 2007, ch. Linear direct connections, pp. 425-436.

[9] J.-L. Loday and D. Quillen, "Cyclic homology and the lie algebra homology of matrices," Comment. Math. Helvetici, vol. 59, pp. 565-591, 1984.

[10] J.-L. Loday, Cyclic Homology, ser. Grundlehren der mathematischen Wissenschaften. Springer, 1992, vol. 301.

[11] G. I. Sharygin, "Local formulae for characteristic classes of a principal $G L_{n}$-bundle," Sbornik Mathematics, vol. 199, pp. 1547-1577, 2008.

[12] N. Teleman, "3-Fold local index theorem," An. Şt. Univ. Ovidius Constanţa, vol. 20, pp. 171-196, 2012.

Author's address

Jan Kubarski

Institute of Mathematics, Lodz University of Technology, Wólczańska 215, 90-924 Łódź, Poland

E-mail address: jan.kubarski@p.lodz.pl 